Estudios de la Paz y el Conflicto

Revista Latinoamericana

IUDPAS-UNAH

Número Especial 1, pp. 43-52

ISSN-e: 2707-8922 / ISSN: 2707-8914

DOI: $10.5377 /$ rlpc.v0i0.9502

Anual 2019

\title{
EDUCAR EN TIEMPOS DE CÓLERA. PEDAGOGÍA PARA LA CONSTRUCCIÓN DE PAZ, RESPETO POR LOS DERECHOS HUMANOS Y DESARME
}

\author{
EDUCATING IN TIMES OF CHOLERA. PEDAGOGY FOR PEACEBUILDING, \\ RESPECT FOR HUMAN RIGHTS AND DISARMAMENT
}

\author{
Alicia Cabezudo \\ International Peace Bureau (IPB) \\ alicia.cabezudo@,ipb.org
}

Cómo citar / citation

Cabezudo, A. (2019). "Educar en tiempos de cólera. Pedagogía para la construcción de paz, respeto por los derechos humanos y desarme", Estudios de la Paz y el Conflicto, Revista

Latinoamericana, Número Especial 1, 43-52. DOI: 10.5377/rlpc.v0i0.9502

\begin{abstract}
Resumen
La educación para la paz y para los Derechos Humanos en búsqueda de la verdad y la justicia es hoy una obligación ética, una necesidad social y un imperativo incuestionable en el campo de la docencia latinoamericana. Para ello es necesario articular sólidos puentes de trabajo y cooperación con la sociedad civil y con las organizaciones no gubernamentales. Es necesario revisar conceptos tradicionales, evaluar prácticas pedagógicas y elaborar proyectos desde cada uno de nuestros espacios de vida y de trabajo - partiendo de la convicción que los esfuerzos para producir transformaciones no son antagónicos sino complementarios. Este artículo intenta demostrar que la Educación para la Paz implica, en realidad, un renovado compromiso con los principios pedagógicos democráticos y un imperativo ético asumirlos concretamente.
\end{abstract}

Palabras clave

Educación; Paz; Derechos Humanos; Ética; Pedagogía democrática.

\begin{abstract}
In the education field of the present time in Latin America education for peace and Human Rights towards truth and justice is an ethic commitment, a social need and a nonquestionable theme. Hence it is necessary to create links in between civil society and the non - governmental organizations building strong bridges of cooperation and work. It is also important to review traditional concepts, to evaluate the education practices and to
\end{abstract}


design projects from our own life, social and working places. Departing from the conviction that the existing efforts for making changes are not antagonistic but complementary. This article intends to demonstrate that Education for Peace implies a permanent compromise with democratic principles in pedagogy and it is an ethic need to assume them in concrete actions and not only in theory.

\section{Keywords}

Education; Peace; Human Rights; Ethic commitment; Democratic pedagogy.

\section{INTRODUCCIÓN}

La paz no sólo se define por la ausencia de guerra y de conflicto, es también un concepto dinámico que necesita ser aprendido en términos positivos. Lo mismo sucede con los Derechos Humanos, los que deben afirmarse con presencia de justicia y solidaridad social, garantizando a todos los seres humanos el derecho a vivir con dignidad a lo largo de toda la vida. Su promoción y enseñanza es fundamental (UNESCO, 1994).

¿Qué tipo de conocimientos se requieren para abordar los retos de cara a la construcción de Paz?

La educación para la paz y el respeto a los Derechos Humanos adquiere en los países latinoamericanos una particular actualidad al contrastar los valores que ella implica con los horrores de la guerra, la violencia y la destrucción que nos conmueven diariamente.

En forma cotidiana, al analizar la desigualdad y la injusticia de nuestra realidad socioeconómica o la violencia brutal de sociedades "modelo" donde los adolescentes masacran a sus maestros y compañeros, donde el peligro acecha a cualquier hora en cualquier calle, donde la convocatoria a la guerra es una práctica habitual para dirimir conflictos internos o internacionales, nos estamos enfrentando ante situaciones de violencia extrema.

Aquí la respuesta del educador, del profesor, maestro, padre o gobernante se vuelve vacía de contenido. Las explicaciones se agotan y la práctica de construcción del conocimiento mediante investigación, lectura, análisis de información, entrevistas, génesis o reflexión sobre los conflictos nos imprime un modelo de práctica educativa relativamente ingenuo donde -en definitiva- aún los mismos educadores quedan insatisfechos.

Pese a su indudable importancia la educación para la Paz y Derechos Humanos -eje didáctico transversal en muchos diseños curriculares internacionales- ha sido concebida como una temática subsidiaria en la selección de contenidos del sistema educativo formal. Eje didáctico necesario pero aleatorio. Importante pero no esencial. Presente pero ausente. "Discurso curricular que ennoblece sin modificar ni concebir alternativas nuevas en la formación ética y ciudadana de docentes y alumnos, siendo un contenido cada vez más necesario en el mundo en que vivimos" (Cabezudo, 2013, 21).

La educación para la paz es hoy un campo pluridimensional e incluye un conjunto de conceptos, ideas y actividades que se demuestran desde las acciones de sensibilización y divulgación para promover una Cultura de Paz, hasta prácticas pedagógicas concretas en el ámbito de la educación formal, no formal e informal. Prácticas cuyos objetivos específicos se vinculan a valores esenciales de la convivencia humana tales como la tolerancia, el respeto y la defensa de los derechos -garantizados por las autoridades del estado en toda sociedad 
democrática. Para Johan Galtung (1997), la educación para la Paz debe articularse desde una perspectiva más amplia que la meramente pedagógica institucionalizada y esta educación debe incluir el estudio, la investigación y la resolución de conflictos por vía pacífica como objetivos fundamentales en un proceso de aprendizaje integral que trasciende el ámbito de la educación formal. Se convierte en un imperativo pedagógico y ético a la luz de los acontecimientos del mundo actual.

El concepto moderno de educación para la paz o para la construcción de Cultura de Paz incluye lo que para nosotros es también educación en Derechos Humanos, donde su significación se amplía y adquiere un sentido concreto y cotidiano vinculado a la vida diaria, a las prácticas sociales, a las políticas de gobierno y a las relaciones interpersonales e interestatales.

Educación en Derechos Humanos no se refiere únicamente a aspectos tradicionales relacionados con las violaciones a los derechos individuales -suspensión de éstos, detención arbitraria, desaparición forzada, tortura, secuestro, muerte- sino que se compromete con el análisis y monitoreo del cumplimiento de estos derechos, así como los derechos sociales, culturales y políticos por parte de los estados nacionales (Monteiro y Tavares, 2010).

Por ello, la revisión pedagógica de viejos conceptos expande en un sentido más amplio las significaciones -contraponiéndose a aquella más limitativa que relegaba el término paz a sinónimo de "ausencia de guerra" o "silencio de los cañones". Se trata de una noción más abarcativa y compleja incluyendo los conceptos de equidad y justicia, dignidad y solidaridad, respeto a los derechos de los pueblos y a la diversidad cultural, lo que nos conduce directamente al campo de los Derechos Humanos.

Es una noción extendida en el mundo pedagógico de hoy, que la educación para la paz debe encarar y desarrollar las demandas más urgentes de la sociedad. En Europa estas demandas se vinculan a la preocupación por el riesgo nuclear, los complejos militares industriales, el desarrollo tecnológico sin controles, la contaminación ambiental, el desarme - "además de aspectos relacionados con problemas concretos de Derechos Humanos, tales como la migración, el desempleo, el racismo y la xenofobia creciente" (Haavelsrud, 2010, 159).

En América Latina la educación para la paz y Derechos Humanos contempla diversos enfoques: tanto aquellos que surgen en particular en regiones que han vivido o viven conflictos bélicos hasta aquellos que la consideran como una respuesta activa de la sociedad civil ante la impunidad y las graves violaciones a los Derechos Humanos acaecidas en las sucesivas dictaduras del continente y aún bajo sistemas reconocidos como "democráticos" o Estados de Derecho.

Para los países de América Latina el problema fundamental radica no sólo en el conflicto militar declarado sino también en la omisión sistemática por parte de los gobiernos democráticos de los derechos políticos, económicos, sociales y culturales, los que no son ni reconocidos ni respetados pese a su status constitucional. Se produce, además, no sólo su omisión sino su violación -aún en aquellos gobiernos que provienen de la votación ciudadana, pero practican el sistemático incumplimiento de los derechos otorgados por la carta constitucional.

Por lo tanto, la acción educativa en América Latina es absolutamente imperativa en lo que respecta a estos temas, donde muchos estados de los tiempos democráticos se han comprometido tibiamente con ellos y donde las organizaciones de la sociedad civil -como en 
muchos otros problemas y temas- se han responsabilizado de su desarrollo y promoción casi en forma excluyente (Cabezudo, 2015).

\section{EL ROL DE LA EDUCACIÓN}

Entendemos que la educación debe sostener y preparar para la vida en una democracia plena -entendida tanto como sistema político como forma de vida- ya que ésta promueve una sociedad en la que son respetados los principios de los Derechos Humanos esenciales y la dignidad de todos los hombres y mujeres, así como la participación cívica y solidaria. Por ello, la justicia social y el respeto a los Derechos Humanos deberían convertirse en objetivos a lograr desde la supra-estructura política, reflejándose en los contenidos curriculares del sistema (Faure, et all, 2005).

La democracia no consiste en un modelo abstracto, sino que involucra procesos complejos al estructurar líneas de pensamiento y comportamientos transferidos a los sistemas educativos formales y a las prácticas de enseñanza en los espacios no formales e informales. Debemos por ello fortalecer la democracia para poder organizar nuevas estrategias y herramientas para la educación para la paz, los Derechos Humanos y el desarme, lo que presupone en primer lugar, los siguientes presupuestos:

- La paz mundial, así como el conocimiento y la defensa de los Derechos Humanos constituyen premisas racionales y objetivos viables para la educación;

- Los educadores deberían demostrar su liderazgo en respaldar los conceptos de paz, dignidad humana y convivencia internacional;

- La justicia, el respeto mutuo y el respeto a las diferencias pueden ser enseñados y aprendidos;

- Los estudiantes y la ciudadanía en su conjunto deben desarrollar pensamiento crítico y ser apoyados en la adquisición de conocimientos que enriquezcan su visión de asuntos y problemáticas nacionales, regionales e internacionales.

- Debe proveerse a toda la población de los conocimientos, las actitudes, la mutua comprensión, las habilidades y los medios necesarios para transformar el continente en una región que solucione los conflictos de forma pacífica y no apoye medidas violentas y contradictorias a los principios que sustentan los Derechos Humanos.

- Debe desarrollarse un proceso de concientización acerca de la violencia de las estructuras socio-económicas en que vivimos, ya que estas estructuras pueden ser tan peligrosas como el conflicto directo en la consecución de la paz y el libre ejercicio de los derechos.

La educación para la paz y los Derechos Humanos debería "ser incluida como un objetivo general en todas las estructuras educativas de nuestros países, como consecuencia de una búsqueda permanente por la justicia, la igualdad y la seguridad para todos los miembros de la sociedad" (Cabezudo y Haavelsrud, 2007, 241).

Es indudable que este tipo de educación otorga un desarrollo integral en el cual la adquisición de valores universales y el conocimiento de los derechos y garantías ciudadanas colaboran para un mayor compromiso con la realidad, el bienestar común y las posibilidades de trabajar como actores dinámicos de transformación y cambio social (Iglesias, 2006).

La presencia de gobiernos democráticos en los países latinoamericanos ha abierto espacios que ponen a prueba nuestra capacidad de desarrollar propuestas educativas favorables a estos Estudios de la Paz y el Conflicto, Revista Latinoamericana, IUDPAS-UNAH, Número Especial 1, 2019, pp. 43-52. DOI: 10.5377/rlpc.v0i0.9502 
objetivos. Por ello la educación para la paz y los Derechos Humanos debe hacer uso de estos espacios disponibles introduciendo sus metas en el sistema educativo formal y en todas las instancias de la educación no formal e informal involucrando diversos niveles del aprendizaje.

Por otra parte, es imposible hablar acerca de este tipo de educación sin superar los métodos autoritarios de la gestión educativa tradicional, adoptando en su lugar ideas de autonomía, responsabilidad y diálogo en los espacios educativos que se generen. Estas premisas implican - sin duda - la responsabilidad del Estado en asegurar una educación que promueva tanto el desarrollo integral del individuo como su inclusión en la sociedad a través de la creación de una "conciencia social abierta al cambio y la participación" (Freire, 1970, 86).

Muchos sistemas educativos latinoamericanos en los últimos años han intentado crear una cultura basada en la libertad y la difusión de los Derechos Humanos, pero quizás han omitido la premisa fundamental acerca de que la libertad significa una participación social en continua expansión, como resultante de un compromiso activo con la realidad. Participación que debe desarrollarse y practicarse también en el proceso educativo.

La enseñanza de principios basados en las ideas de desmilitarización, seguridad humana, Derechos Humanos, desarrollo y cooperación internacional provee de herramientas inestimables en la construcción de una cultura de paz, las que posibilitan un análisis del mundo pluridimensional e integral y posibilita la planificación de proyectos para mejorarlo y modificarlo en el sentido que aquí estamos refiriéndonos.

La enseñanza de la paz, los Derechos Humanos y el desarme tiene además en América Latina una particular relevancia en respuesta a la historia de violaciones sistemáticas producidas a partir del establecimiento de gobiernos antidemocráticos y dictaduras militares, particularmente durante fines de la década de los '70 y durante toda la década del 80'. Asimismo, la inclusión de nuevas técnicas de mediación y resolución de conflictos por vía pacífica, el avance del derecho internacional y de la influencia de los movimientos sociales no violentos en la conciencia de los pueblos actúan como elementos de presión, creando mayor sensibilización e información acerca de los problemas que nos ocupan y promoviendo cambios en la legislación de los países latinoamericanos en sus programas educativos.

Estos factores esenciales allanaron el camino para la introducción de temas específicos vinculantes a los conceptos de Paz, Desarme, Derechos Humanos, Derechos del Niño, Género, Diversidad y Multiculturalidad, Sustentabilidad y Desarrollo, Desmilitarización y Desarme, Mediación y Resolución de Conflictos en muchos sistemas educativos y programas de educación para la ciudadanía a partir de la década del 90' del siglo pasado (Lederach, 2006)

Por otra parte, en el proceso de consolidación de los sistemas democráticos en Latinoamérica los sistemas educativos y programas no formales no sólo deberían promover estos conceptos y principios sino desarrollar la posibilidad de su implementación a través de acciones concretas en y con la comunidad.

De esta manera las prácticas educativas operarían como un vínculo entre el sistema jurídico formal existente -a nivel nacional, regional e internacional-y las experiencias de la vida cotidiana. Si el objetivo de la educación es la preparación para la vida, promoviendo una actitud crítica y transformadora de quienes estudian, es fundamental la sensibilización acerca de la validez de la paz y la solidaridad en el seno de la sociedad para el desarrollo del bien común. 
Esta sensibilización debe ser obtenida en forma práctica y operativa, a través de un análisis crítico de la realidad que nos rodea, planificando acciones concretas a través de proyectos pedagógicos con la comunidad, los vecinos y las organizaciones no gubernamentales.

Por práctica y operativa entendemos que quienes estudian y aprenden (alumnos, ciudadanos, población en general) deben tener un rol activo al trabajar estos temas vinculándolos con sus actividades diarias, aprendiendo y practicando sus derechos desde la práctica cotidiana y compartiendo esta experiencia y la adquisición de nuevos conocimientos en forma colectiva con los demás actores del aprendizaje.

Aprendizaje que se convierte en un saber y una práctica social de extraordinaria importancia ya que puede ser transferida a nuevas situaciones problemáticas para buscar soluciones posibles desde una perspectiva individual o colectiva.

\section{UN ABORDAJE PRÁCTICO}

Fundamentalmente, el objetivo de esta propuesta se basa en hacer que los problemas concernientes a la paz, el desarme y los derechos humanos se conozcan y aborden a través del estudio de cuestiones emergentes de la propia realidad (realidad micro) la que luego deben vincularse a realidades cada vez más complejas y lejanas (análisis macro) en un eje temporal analizado desde el presente, pero con fuerte raíces en el pasado y perspectiva de visión futura (Haavelsrud, 2010).

Análisis y búsqueda que tiende a la concientización acerca de las características del mundo en que vivimos y la búsqueda de alternativas de cambio desde nuestra participación individual y colectiva para construir un mundo más justo, equitativo y pacífico. Un mundo en el cual la resolución de conflictos por vía pacífica, el diálogo constructivo, la participación y la solidaridad sean a su vez sistemáticos mecanismos de transformación hacia una sociedad más democrática.

En realidad, la educación basada en estos principios debería ser una política de Estado y no sólo un objetivo específico para organizar la enseñanza.

La propia comunidad educativa, así como todas las áreas del conocimiento reflejadas en diseño curriculares específicos permiten la incorporación de principios vinculados a la Paz, el desarme y los Derechos Humanos. Esta incorporación debería desarrollarse en un proceso de interacción constante, como contenido, como un criterio hacia la construcción compartida del conocimiento, como un eje para actividades conjuntas entre la escuela, la sociedad y las instituciones.

Sin duda el sistema educativo formal - que es parte del Estado - debe responsabilizarse por la promoción, enseñanza y práctica de una educación sistemática por la paz, los Derechos Humanos y el desarme desde una perspectiva interdisciplinaria y transversal en toda planificación curricular e institucional. Proceso que conlleva el estudio y planificación de un programa de políticas educativas al que se proponen las siguientes características:

- Ser progresivo, es decir, evolutivo, lento y profundo, teniendo en cuenta los conflictos sociales potenciales y sus resoluciones;

- Ser sistemático, coherente con un propósito político;

- Ser global, acompañando a las políticas públicas desarrolladas en otros ámbitos de la sociedad; 
- Debe implicar la recuperación de las identidades históricas y culturales, que permiten a las personas ser protagonistas de la historia y actores clave de transformaciones en su propia realidad;

- Debe permitir modificaciones y cambios en las estructuras y en los modos de vida de la sociedad, aplicando perspectivas multiculturales e integradoras.

El abordaje geográfico e histórico de temas es necesario, pero no suficiente. Tampoco el aprendizaje aislado de conceptos tales como nacionalismos, soberanía y Estado. Temáticas fundamentales como la realidad de los diferentes grupos sociales de la región y su problemática convivencia; las soluciones y los desencuentros ante los conflictos; la situación de los refugiados y su desamparo ante los ataques de "amigos" y enemigos; el armamentismo y la venta de armas como rentable negocio mundial, la violación sistemática de Derechos Humanos deben "extenderse pedagógicamente" en un alcance mayor que el mero conocimiento.

Extenderse pedagógicamente significa que los temas deben ser objeto de reflexión, discusión, investigación y crítica. En un ejercicio permanente de búsqueda de la verdad, de intercambio de opiniones, de aprendizajes individuales y colectivos, en donde se discuten puntos de vista diversos y se aprende a respetar a todos aquellos que piensan diferente y en forma constructiva para todos.

La desigualdad social, la carencia de recursos vitales por parte de gran número de la población latinoamericana, el desempleo y la miseria crean desesperanza y desconfianza en los gobiernos elegidos democráticamente en toda la región. Y es verdad que mecanismos autoritarios, control de información, paternalismos y cacicazgos, crímenes e impunidad, forman parte de nuestra vida política.

En este sentido, la guerra no está tan lejos - y no sólo en términos de conflicto armado.

En los países de nuestra región es una guerra cotidiana vivir en pésimas condiciones de salud, educación, empleo y vivienda, menoscabándose diariamente principios básicos inherentes a la dignidad humana de gran parte de la población.

La tarea educativa debe obligatoriamente promover un análisis integral de la violencia y la injusticia para posibilitar una reflexión crítica y transformadora que genere cambios reales y posibles. Y esta reflexión debe ser realizada por la acción educativa pero también por los gobiernos y la ciudadanía toda.

De acuerdo a esta propuesta los temas vinculantes a la educación para la Paz y los Derechos Humanos se desarrollan en el formato de núcleos significativos desde las diferentes áreas y disciplinas curriculares o desde los diversos campos disciplinares y prácticas sociales, basándose en el concepto holístico de que no existe ninguna situación de paz y Derechos Humanos ajena a cualquier tipo de conocimiento.

Se propone la organización de ejes problemáticos donde aparecen los temas fundamentales a desarrollar, la bibliografía básica, los recursos potenciales y las actividades que conducen al aprendizaje. Al mismo tiempo, estos ejes problemáticos no se presentan como estructuras impuestas sino como alternativas posibles dentro de un tema que es discutido y seleccionado dentro de un proceso de trabajo conjunto y participativo entre todos los actores del aprendizaje donde éste se desarrolle - sea en el sistema formal o en los diversos espacios no formales.

En toda América Latina, a partir del restablecimiento de las democracias a mediados de los años 80', los estados crearon asignaturas denominadas de diferentes formas -Educación 
Democrática, Educación Cívica, Instrucción Cívica, Formación Ciudadana, Educación para la Ciudadanía, Conocimiento de la Realidad, Formación Ética y Ciudadana, etc- las que se consideraron plataformas integradoras para la implementación de este tipo de contenidos ante una necesidad urgente de innovación ideológica-pedagógica después de las pavorosas dictaduras (ONU, 1999 y UNESCO 1994).

Sus contenidos curriculares tuvieron como objetivo específico la adquisición rápida y concreta de valores y normas de comportamiento "deseables" y "necesarias" en incipientes sociedades democráticas de transición, tendientes a consolidar un estilo de vida y una percepción crítica de la realidad político-social que debía ser reconstruida después de las experiencias totalitarias en prácticamente todo el continente.

Campos disciplinares como Historia, Literatura, Artes y Economía actuaron como asignaturas de apoyo en un intento de promover un cambio basado en nuevas corrientes pedagógicas y metodológicas, así como en renovados paradigmas científicos y contenidos innovadores que eran considerados necesarios para fortalecer la formación sistemática en las incipientes democracias.

Se generalizó también la introducción de la comunidad en la tarea pedagógica asociaciones de la sociedad civil, Organizaciones No Gubernamentales, proyectos del gobierno local e Iglesias- y se profundizó la importancia de los aprendizajes en el sistema no formal -más abierto, flexible y operativo que el sistema fuertemente jerarquizado de la educación formal. Se generalizó el aprendizaje por proyectos sobre problemáticas de la realidad cercana conducente a establecer un contacto directo con el objeto de estudio y sus evidentes ventajas: clara detección de problemas, búsqueda de alternativas válidas y puesta en práctica de acciones con el objetivo de resolver el problema inicial.

En realidad, la educación para la Paz y Derechos Humanos no tendría ningún sentido sin la ejecución de actividades que apelen a la organización de este tipo de prácticas educativas en los que los sujetos de aprendizaje asumen un activo rol como agentes colaboradores y transformadores "durante y desde" el mismo proceso.

Así, el aprendizaje tradicional atraviesa las paredes de la escuela, de los institutos, de las universidades y de la Academia, contribuyendo a la sensibilización de la opinión pública y mejorando las relaciones humanas mediante el diálogo y la participación colectiva en actividades pedagógicas de significación social (Martínez, 2001).

Estas prácticas vinculada y asociadas al campo de la construcción de cultura de paz y Derechos Humanos deberían presentar condiciones básicas tales como:

- Ser experiencias colectivas dentro de un marco de igualdad en las relaciones grupales y con el coordinador de grupo (maestro, profesor, líder social, etc)

- Ser prácticas concretas en resolución de problemas y no simplemente estudio de datos.

- Estar basadas en una relación educador/educando esencialmente horizontal y democrática, representando o anticipando las relaciones democráticas en la sociedad.

- Facilitar la construcción de una conciencia democrática y pluralista, defendiendo los principios de la paz, el desarme y la validez de los fundamentos constitucionales de un Estado de Derechos caracterizado por la observancia y vigencia de los Derechos Humanos. 


\section{CONCLUSIÓN}

La educación como constructora de Paz, defensora de los Derechos Humanos y promotora del Desarme es hoy una obligación ética, una necesidad social y un imperativo incuestionable en el campo de la docencia latinoamericana.

Para ello es necesario articular sólidos puentes de trabajo y cooperación con la sociedad civil y con las organizaciones no gubernamentales.

Es urgente también revisar conceptos tradicionales, evaluar nuevas prácticas, elaborar proyectos desde cada uno de los espacios de vida y de trabajo - partiendo de la convicción de que los esfuerzos individuales y colectivos para producir cambios y transformaciones no son antagónicos sino complementarios.

La educación por la Paz y los Derechos Humanos es además un desafío para todos los que desean una sociedad más justa, equitativa y solidaria en el contexto actual del continente. Lo que implica un renovado compromiso con principios pedagógicos democráticos y participativos, así como es un imperativo ético la tarea de asumirlos.

Construir y promover la Educación por la Paz, los Derechos Humanos y el Desarme en América Latina, requiere una educación que no sólo apunte a conocimientos, sino que habilite para la acción, para el desarrollo de un pensamiento crítico, para la adquisición de métodos de reflexión intelectual y de trabajo colectivo.

Una educación que colabore en la búsqueda de una transformación social y política mediante métodos no violentos y el respeto de culturas diversas. Una educación adecuada y comprometida con las necesidades y anhelos del continente en que habitamos.

He aquí el gran desafío pedagógico del siglo XXI en el campo pedagógico y en la tarea educadora diaria de América Latina.

\section{REFERENCIAS}

Cabezudo, A. (2013) “Acerca de una educación para la paz, los derechos humanos y el desarme", Educação, $36 \quad$ (1), 44-49. (Disponible online: http://revistaseletronicas.pucrs.br/ojs/index.php/faced/article/view/12313/8739)

Cabezudo, A. (2015). "Educación de las comunidades en el espacio de la Ciudad Educadora", Revista TAREA, 90, 28-33. (Disponible en: http://tarea.org.pe/wpcontent/uploads/2016/04/Tarea90_28_Alicia_Cabezudo.pdf)

Cabezudo, A. y Haavelsrud, M. (2007). Rethinking Peace Education. En Webel, C. y Galtung, J. (Eds.) Handbook of Peace and Conflict Studies. London: Routledge/Taylor and Francis Book Ltd.

Faure, E., Herrera, F., Kaddoura, A.R., Lopes, H., Petrovsky, A, Rahnema, M., y Champion Ward, F. (2005). Aprender a Ser. Madrid: Ediciones UNESCO - Editorial Alianza.

Freire, P. (1970). Pedagogía del Oprimido. México: Siglo XXI Editores.

Galtung, J. (1997). Manual para el entrenamiento de Programas de las Naciones Unidas. NYC: Editorial Naciones Unidas,.

Haavelsrud, M. (2010). El poder de la Educación. Aprendizajes para vivir en libertad. Tromso: Arena. 
Iglesias, C. (2006). Educar pacificando. Una pedagogía de los conflictos. Santiago de Compostela: Cultura de Paz.

Lederach, J.P. (2006). Educar para la Paz. Barcelona: Fontamara.

Martínez, V. (2001). Filosofía para hacer las Paces. Barcelona: Icaria.

Monteiro, A. y Tavares, C. (2010). El papel de la Educación en Derechos Humanos en la formación de la Ciudadanía Activa. Pernambuco: Cortez.

ONU (Organización de Naciones Unidas) (1999). Resolución A/RES/53/243 - Declaración y Programa de Acción sobre una Cultura de Paz. New York: ONU.

UNESCO (Organización de las Naciones Unidas para la Educación, la Ciencia y la Cultura) (1994). Reunión Consultiva del Programa Cultura de Paz.

\section{REFERENCIA DE LA AUTORA}

Alicia Cabezudo: Doctora en Pedagogía, especialidad en Historia y Ciencias Sociales, Universidad de Ginebra, y Maestría en Educación en Ciencias Sociales con especialidad Educación para los DDHH y la Democracia en América Latina, Universidad de Barcelona. Su área de especialización es Educación para la Paz, Democracia, Ciudadanía, Resolución de Conflictos y Derechos Humanos, temas sobre los cuales desarrolla actividades de formación, investigación y enseñanza. Se ha especializado en los últimos veinte años en formación de educadores, líderes sociales y servidores públicos. Ha sido Coordinadora en Argentina del Proyecto Educación para la Democracia, Cultura de Paz y Derechos Humanos de la Fundación Cultura de Paz, presidida por el Dr. Federico Mayor Zaragoza y Directora Organizadora de la Red Internacional de Ciudades Educadoras para América Latina, con base en el Ayuntamiento de Barcelona (España) y la Municipalidad de Rosario (Argentina).

Recibido: 29-01-2020

Aceptado: 09-02-2020

(c) (i) Licencia Creative Commons Reconocimiento 4.0 (CC BY 4.0).

Estudios de la Paz y el Conflicto, Revista Latinoamericana, IUDPAS-UNAH, Número Especial 1, 2019, pp. 43-52. DOI: 10.5377/rlpc.v0i0.9502 